\title{
Blockade of intercellular adhesion molecule-1 prevents angiotensin II-induced hypertension and vascular dysfunction
}

\author{
Ping-Ping Lang ${ }^{1} \cdot$ Jie Bai $^{1} \cdot$ Yun-Long Zhang ${ }^{1} \cdot$ Xiao-Lei Yang ${ }^{1} \cdot$ Yun-Long Xia ${ }^{1} \cdot$ Qiu-Yue Lin ${ }^{1} \cdot$ Hui-Hua Li ${ }^{1}$
}

Received: 17 May 2019 / Revised: 3 August 2019 / Accepted: 6 August 2019 / Published online: 16 September 2019

(c) The Author(s), under exclusive licence to United States and Canadian Academy of Pathology 2019

\begin{abstract}
Monocyte and adhesion infiltration into the arterial subendothelium are initial steps in hypertension development. The endothelial intercellular adhesion molecule-1 (ICAM-1) has been implicated in the recruitment and adhesion of leukocytes in several cardiac diseases. However, the role of ICAM-1 in angiotensin II (Ang II)-induced hypertension development remains unknown. Hypertension was induced by administering an infusion of Ang II (1000 ng/kg/min) to wild-type (WT) mice treated with an IgG control or ICAM-1 neutralizing antibody (1 and $2 \mathrm{mg} / \mathrm{mouse} /$ day, respectively). Blood pressure was determined using the tail-cuff system. Vascular remodeling was assessed by performing a histological examination. Inflammation and reactive oxygen species (ROS) levels were determined by using immunostaining. Vascular dysfunction was assessed by aortic ring assay. The expression of fibrotic markers, cytokines and NOX was evaluated by quantitative realtime PCR analysis. Our results demonstrate that Ang II infusion markedly increased the ICAM-1 level in the aorta. Blocking ICAM-1 with a neutralizing antibody significantly attenuated Ang II-induced arterial hypertension, vascular hypertrophy, fibrosis, macrophage infiltration, and ROS production and improved vascular relaxation. In conclusion, ICAM-1-mediated monocyte adhesion and migration play a critical role in Ang II-induced arterial hypertension and vascular dysfunction. ICAM-1 inhibitors may represent a new therapeutic strategy for the treatment of this disease.
\end{abstract}

\section{Introduction}

Hypertension is a risk factor for cardiovascular diseases, including cardiac hypertrophy, myocardial infarction, and heart failure (HF) [1]. Hypertension-induced hormonal disorder promotes vascular hypertrophy, which increases peripheral vascular resistance and endothelial dysfunction, thereby contributing to impaired microcirculation [2]. The renin-angiotensin system (RAS) plays a critical role in the development of hypertension. Angiotensin II (Ang II), the main effector hormone of the RAS, triggers the

These authors contributed equally: Ping-Ping Lang, Jie Bai

Qiu-Yue Lin

lqy8986@163.com

$\triangle$ Hui-Hua Li

hhli1935@aliyun.com

1 Department of Cardiology, Institute of Cardiovascular Diseases, First Affiliated Hospital of Dalian Medical University, No.193, Lianhe Road, Xigang District, 116011 Dalian, China inflammatory response and oxidative stress, resulting in structural or functional changes in the vasculature $[3,4]$. Notably, Ang II mediates the migration and infiltration of proinflammatory cells into the vasculature and other tissues by inducing the expression of adhesion molecules and chemokines [5, 6]. Among these cells, monocytes/macrophages are crucial for hypertension and vascular dysfunction induced by Ang II or DOCA-salt [3, 4]. As shown in our recent study, chemokines and their respective receptors, particularly the CXCL1-CXCR2 signaling pathway, play critical roles in mediating the migration of monocytes/ macrophages into vessels and in the development of hypertension [3]. However, the precise mechanisms by which Ang II promotes monocyte adhesion to endothelial cells (EVs) and the subsequent recruitment steps remain to be elucidated.

Cell-adhesion molecules (CAMs) are a subset of celladhesion proteins located on the cell surface that play key roles in allowing cells to bind to other cells or with extracellular matrix molecules. Four major super families of CAMs have been identified: integrins, the immunoglobulin superfamily (IgSF), cadherins, and selectins [7]. The IgSF is one of the largest super families of proteins in the body, and 
it includes many diverse CAMs, such as intercellular celladhesion molecules (ICAMs) and vascular cell-adhesion molecules (VCAMs), which undergo heterophilic binding with molecules such as carbohydrates and integrins [8]. Interestingly, both ICAMs and VCAMs are expressed on vascular EVs and interact with integrins such as CD11/18 on leukocytes to assist with leukocyte adhesion and trafficking $[8,9]$. Five members of the ICAM immunoglobulin superfamily have been identified: ICAM-1 to 5. ICAM-1 is a major adhesion receptor for monocyte/macrophage attachment to EVs at inflammatory sites through binding to its receptors LFA-1 and Mac-1 [10-12]. Moreover, ICAM1 expression is induced by various inflammatory stimuli [11]. An TNF- $\alpha$ treatment markedly upregulates ICAM-1 expression on the surface of neutrophils in the perfused heart [13]. Pressure overload induces ICAM-1 expression in ECs of intramyocardial arterioles [14]. Furthermore, leukocyte-depleted reperfusion attenuates ICAM-1 expression and cardiac dysfunction [15]. However, the role of ICAM-1 in the Ang II-induced development of hypertension and vascular dysfunction remains unclear.

As shown in the present study, ICAM-1 plays a critical role in Ang II-induced hypertension and vascular dysfunction. The levels of the ICAM-1 mRNA and protein were significantly upregulated in Ang II-infused aortas compared with the saline control. Conversely, blocking ICAM-1 with a neutralizing antibody markedly reduced Ang II-induced arterial hypertension, vascular remodeling, macrophage infiltration into the vascular wall, and ROS production and improved vascular dysfunction. Therefore, these results provide novel evidence that the inhibition of ICAM- 1 exerts a protective effect on hypertension development and represents a new therapeutic target for hypertension.

\section{Materials and methods}

\section{Animals and treatment}

C57BL/6 J wild-type (WT) mice were purchased from the Jackson Laboratory (Sacramento, CA). Male mice (8 weeks old; $n=6$ animals per group) were infused with saline or Ang II at a dose of $1000 \mathrm{ng} / \mathrm{kg} / \mathrm{min}$ (Aladdin, CA) with an osmotic minipump (Alzet MODEL 1007D for 7 days; Alzet MODEL 1002 for 14 days; DURECT, Cupertino, CA) as previously described [3]. A control IgG (A7028, Beyotime, CA) or anti-ICAM-1 neutralizing antibody (BE0020-1, Bio $\mathrm{X}$ Cell, USA) was administered every 2 days beginning 1 day before the operation to block soluble ICAM-1 based on our preliminary results and previous publications [16]. The ICAM-1 neutralizing antibody was administrated by tail intravenous injection (IV.). All mice were maintained under specific pathogen-free conditions and provided free access to a normal diet ( $0.3 \%$ sodium chloride). After the study period, the mice were anesthetized, and the serum was collected and aortic tissues were removed and prepared for further molecular and histological examinations. All procedures were approved by the Institutional Animal Care and Use Committee of Dalian Medical University and conformed to the US National Institute of Health's Guide for the Care and Use of Laboratory Animals.

\section{Blood pressure measurement}

Blood pressure was measured in conscious mice using the tail-cuff method (Softron BP-98A, Tokyo, Japan) as described previously $[3,17]$. The mice were handled gently and not forced to enter the restrainer, and the ambient temperature was maintained at a warm room temperature $\left(25-30{ }^{\circ} \mathrm{C}\right)$.

\section{Enzyme-linked immunosorbent assay (ELISA)}

The level of ICAM-1 in serum was analysed with an ELISA according to the manufacturer's instructions (E-ELM0710c, Elabscience Biotechnology, CA).

\section{Histopathological analysis}

Fragments of the aorta were fixed with $10 \%$ formalin, embedded in paraffin, sectioned at a thickness of $5 \mu \mathrm{m}$, and then routinely stained with haematoxylin and eosin (H\&E) or Masson's trichrome reagent as previously described $[3,17]$. Immunohistochemistry was performed on the aortic sections with an antibody against ICAM-1 (10020-1-AP, Proteintech, CA). Briefly, the aortic sections were permeabilized with $0.3 \%$ Triton X-100/PBS and then incubated with an antibody against Mac-2 (SAB4501746, Sigma, CA) overnight at $4{ }^{\circ} \mathrm{C}$, followed by an incubation with DAPI (1:10000, Sigma) for $5 \mathrm{~min}$ at room temperature. The aortic sections were also stained with dihydroethidium (DHE, 1 $\mu \mathrm{M}$ in PBS) for $30 \mathrm{~min}$ at $37^{\circ} \mathrm{C}$ as described in a previous study [3]. Red fluorescence was detected using fluorescence microscope (OLYMPUS, BX53, Japan).

\section{Quantitative real-time PCR analysis}

Total RNA was isolated from the aorta using TRIzol (Invitrogen, New York, NY) according to the manufacturer's instructions. The first-strand cDNA (1-2 $\mu \mathrm{g})$ templates were synthesized with Superscript II (Invitrogen, New York, NY). Levels of the ICAM-1, IL-1 $\beta$, IL-6, NOX2, NOX-4, collagen I, and collagen III transcripts were detected by quantitative real-time polymerase chain reaction (PCR) analysis with SYBR Mix (Takara, Japan) as previously described [18]. The data were normalized to the 
expression of the GAPDH gene. The following primers were used for the PCR analysis. ICAM-1, forward 5'-GCC TTG GTA GAG GTG ACT GAG-3', and reverse 5'-GAC CGG AGC TGA AAA GTT GTA-3'; IL-1 $\beta$, forward 5'-TG C CAC CTT TTG ACA GTG ATG- $3^{\prime}$, and reverse $5^{\prime}$-TTC TTG TGA CCC TGA GCG AC-3'; IL-6: forward 5'-TTC CAT CCA GTT GCC TTC TTG- $3^{\prime}$, and reverse $5^{\prime}$-TTG GGA GTG GTA TCC TCT TGT GA-3'; NOX-2: forward 5'-CTT CTT GGG TCA GCA CTG GC-3', and reverse 5'GCA GCA AGA TCA GCA TGC AG-3'; NOX-4 forward 5'-GCA CGC CTT CAA CAC CAA G-3', and reverse 5'GCG AGG CAG GAG AGT CAG TG-3', collagen I: forward 5'-TGA CTG GAA GAG CGG AGA GTA C-3', and reverse 5'-TTC GGG CTG ATG TAC CAG TTC- ${ }^{\prime}$ and collagen III: forward $5^{\prime}$-AAA TTC TGC CAC CCC GAA CT-3', and reverse: 5'-CCA GTG CTT ACG TGG GAC AGT-3'.

\section{Vascular relaxation examinations}

The thoracic aortas were isolated, cut into $4 \mathrm{~mm}$ segments and gently mounted on force transducers (Danish Myo Technology, DK) in organ chambers. After stimulation with noradrenaline, the endothelium-dependent or -independent relaxation in response to increasing concentrations of acetylcholine (ACh) or sodium nitroprusside (SNP) was recorded using previously described methods [4].

\section{Cell-adhesion assays}

Human umbilical vein endothelial cells (HUVECs) were isolated from umbilical cords and cultured in endothelial cell medium supplemented with 5\% fetal bovine serum as previously described [3]. Bone marrow-derived macrophages were isolated from the tibias and femurs of WT C57/ BL6 mice (8 weeks old) [3].

Confluent HUVECs were pretreated with Ang II $(100 \mathrm{nM})$ or saline for $24 \mathrm{~h}$ and then treated with the isotype control IgG or anti-ICAM-1 neutralizing antibody for $4 \mathrm{~h}$. The isolated macrophages were labeled with the PKH-2 fluorescent green dye according to the manufacturer's instructions (Sigma-Aldrich, St. Louis, MO) and then added to HUVECs at a macrophage-to-EV ratio of 10:1. After $1 \mathrm{~h}$ of incubation at $37^{\circ} \mathrm{C}$, non-adherent cells were washed with PBS three times and then fixed with $4 \%$ paraformaldehyde. Adherent cells were counted in six randomly chosen fields using a fluorescence microscope (OLYMPUS, IX73, Japan) [19].

\section{Cell migration assays}

Confluent HUVECs were exposed to Ang II $(100 \mathrm{nM})$. The isolated macrophages $\left(6 \times 10^{4}\right)$ were added to the upper chambers of transwell inserts in 24-well tissue culture plates ( $5 \mu \mathrm{m}$ pore size, Corning, New York, USA), and conditioned supernatants from HUVECs were added to the lower wells. After $24 \mathrm{~h}$ of incubation, macrophages that had migrated to the lower chamber were counted in six randomly chosen fields using an inverted microscope (OLYMPUS, IX73, Japan) [19].

\section{Statistical analysis}

All data were analysed using SPSS 19.0 software. Data were compared using one-way ANOVA (Student-Newman-Keuls and Bonferroni tests), independent $t$ test, or chi-square test as appropriate. $P<0.05$ was considered statistically significant.

\section{Results}

\section{Angiotensin II increases ICAM-1 levels in the aorta and serum}

We first examined the expression of ICAM-1 in the aorta 14 days after the Ang II infusion to investigate the regulatory role of ICAM-1 in hypertension. The qPCR analysis revealed a significant increase in ICAM-1 expression in the Ang II-infused aorta at days 7 and 14 (Fig. 1a). Immunohistochemical staining further confirmed that the aortic expression of ICAM-1 was increased after the Ang II infusion in a time-dependent manner (Fig. 1b). Moreover, an ELISA revealed higher serum ICAM-1 levels in Ang IItreated aortas than in saline control-treated aortas at days 7 and 14 (Fig. 1c). Overall, the Ang II infusion increases the ICAM-1 level in the aorta and serum, and ICAM-1 may play a role in hypertension development.

\section{The administration of an ICAM-1 neutralizing antibody attenuates Ang II-induced hypertension, vascular hypertrophy, and fibrosis}

WT mice were treated with an IgG control or ICAM-1 neutralizing antibody ( 1 and $2 \mathrm{mg} / \mathrm{mouse} /$ day, respectively) and Ang II (1000 ng/kg/min) for 7 and 14 days to determine whether ICAM-1 blockade reduced Ang II-induced hypertension. The ELISA revealed dose-dependent decreases in soluble ICAM-1 levels in the blood of mice administered the ICAM-1 neutralizing antibody compared with the IgGtreated group at days 7 and 14, respectively (Fig. 2a). Moreover, the Ang II infusion significantly elevated the blood pressure compared with the saline control, and this effect was markedly attenuated by the ICAM-1 neutralizing antibody in a dose-dependent manner compared with IgGtreated mice (Fig. 2b). A difference in blood pressure was 
Fig. 1 An Ang II infusion upregulates ICAM-1 expression in the aorta. a WT mouse was infused with Ang II (1000 ng/kg/ $\min )$ or saline for 7 or 14 days. Quantitative PCR analysis of ICAM-1 mRNA levels in the aorta. b Images of immunohistochemical staining showing the ICAM-1 positive cells in aortic sections (upper panels). Scale bar, $50 \mu \mathrm{m}$.

ICAM-1 positive area in aortic sections (lower panels, $n=6$ animals per group). c ELISA results for the serum ICAM1 level
A
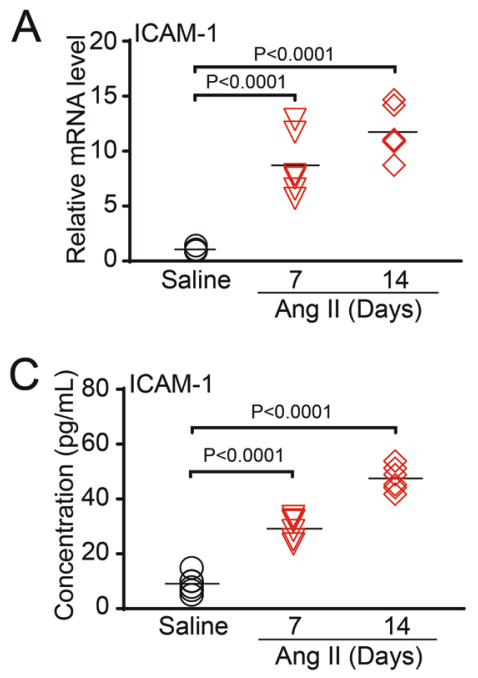

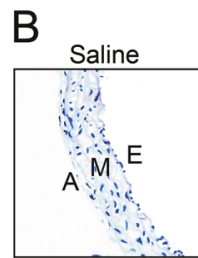

Ang II (Days)
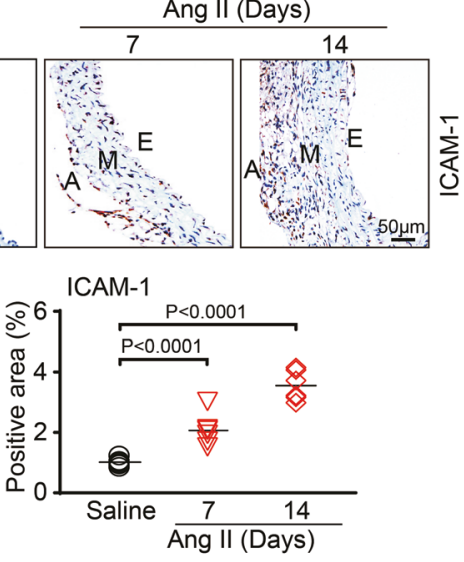

not observed between anti-ICAM-1-treated mice and IgGtreated animals after the saline infusion (Fig. 2b).

Next, we analysed the effect of ICAM-1 inhibition on Ang II-induced vascular hypertrophy and fibrosis by performing H\&E and Masson's trichrome staining. The Ang II infusion led to a significant increase in the aortic wall thickness and collagen deposition in IgG-treated mice, changes that were attenuated in ICAM-1 antibody-treated animals in a dose-dependent manner (Fig. 3a, b). Lower levels of the fibrotic markers collagen I and collagen III were also observed in anti-ICAM-1-treated mice than in IgG-treated animals after the Ang II infusion (Fig. 3c).

\section{Inhibition of ICAM-1 reduces macrophage recruitment and oxidative stress}

We performed immunostaining with a Mac-2 antibody to examine whether ICAM-1 blockade decreases macrophage accumulation in the aorta, Ang II infusion for 14 days significantly increased the number of Mac- $2^{+}$macrophages in the aortas from IgG-treated mice compared with saline control-treated mice (Fig. 4a). In contrast, the number of macrophages was reduced in anti-ICAM-1-treated mice (Fig. 3a). Ang II also increased intracellular ROS production. We then performed DHE staining and found that the Ang II infusion-induced increases in ROS levels in the aorta were abrogated in anti-ICAM-1-treated mice (Fig. 4b). As expected, the Ang II-induced upregulation of IL-1 $\beta$, IL-6, NOX-2, and NOX-4 expression in the aorta was also dosedependently inhibited in anti-ICAM-1-treated mice compared with the IgG-treated group (Fig. 4c). Significant differences in the number of macrophages and ROS production were not observed between the two groups after the saline infusion (Fig. $4 \mathrm{a}-\mathrm{c}$ ). Based on these results,
ICAM-1 promotes Ang II-induced inflammation and oxidative stress in the aorta.

\section{Blockade of ICAM-1 with a neutralizing antibody improves vascular dysfunction}

Inflammation and oxidative stress contribute to Ang IIinduced vascular dysfunction. We next examined whether the inhibition of ICAM-1 ameliorates vascular dysfunction by constructing concentration-relaxation curves in response to ACh or SNP. The Ang II infusion significantly impaired endothelium-dependent vasodilatation in response to $\mathrm{ACh}$ compared with the saline control, whereas vasodilatation was markedly preserved in anti-ICAM-1 mice (Fig. 5a). The Ang II infusion only reduced endothelium-independent vasodilatation in response to SNP in WT mice, and a significant difference was not observed between Ang II-treated mice and saline control mice (Fig. 5b). Thus, the inhibition of ICAM-1 prevents Ang II-induced endothelial dysfunction.

\section{Inhibition of ICAM-1 blocks macrophage adhesion and migration}

Adhesion molecules are essential for leukocyte adhesion to vascular EVs after various stimuli [20]. We analysed the effect of ICAM-1 on macrophage adhesion and migration to further explore the mechanisms by which ICAM-1 inhibition reduces HUVEC dysfunction. The Ang II treatment $(100 \mathrm{nM})$ clearly increased macrophage adhesion to ECs and macrophage migration, and this increase was markedly attenuated by the anti-ICAM-1 antibody (Fig. 6a, b). Therefore, ICAM-1 directly influences macrophage adhesion and migration in vitro. 
A

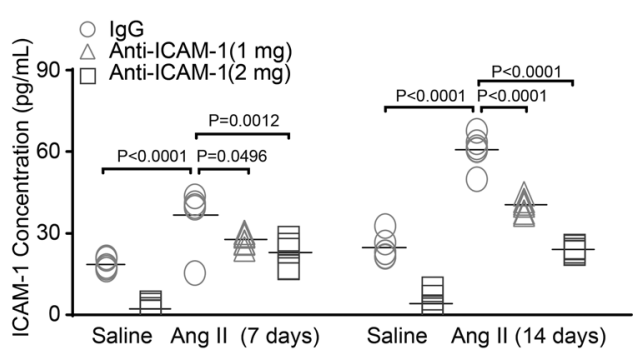

Fig. 2 The administration of an anti-ICAM-1 neutralizing antibody prevents Ang II-induced hypertension. a WT mouse was injected with an anti-ICAM-1 neutralizing antibody ( 1 or $2 \mathrm{mg} / \mathrm{mouse} /$ day) or control IgG every two days beginning 1 day before the Ang II (1000
$B$

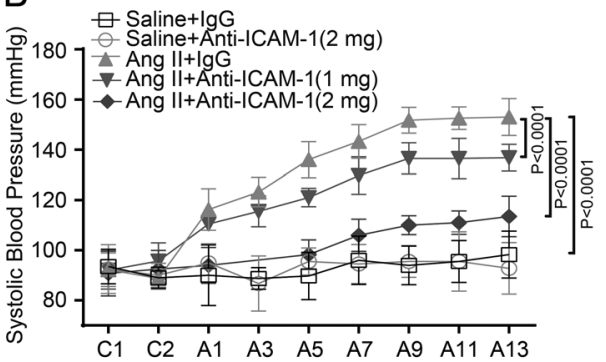

$\mathrm{ng} / \mathrm{kg} / \mathrm{min}$ ) or saline infusion for 7 and 14 days. ELISA results for serum ICAM-1 levels in each group. b An average systolic blood pressure of each group

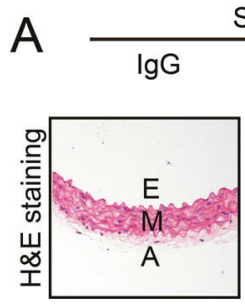

Saline

Anti-ICAM-1

(2 mg)
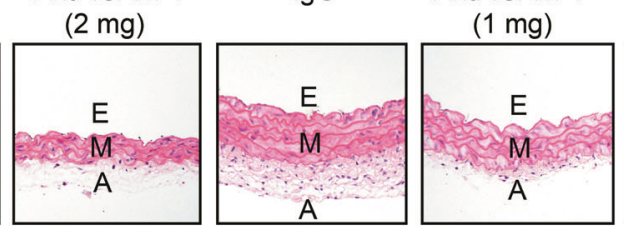

Ang II

Anti-ICAM-1

(2 mg)

B
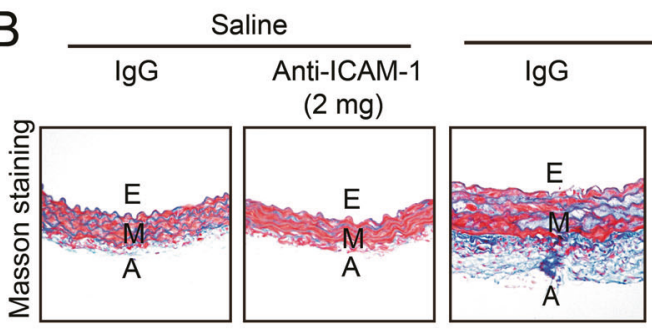

Ang II

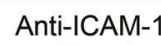

(1 mg)
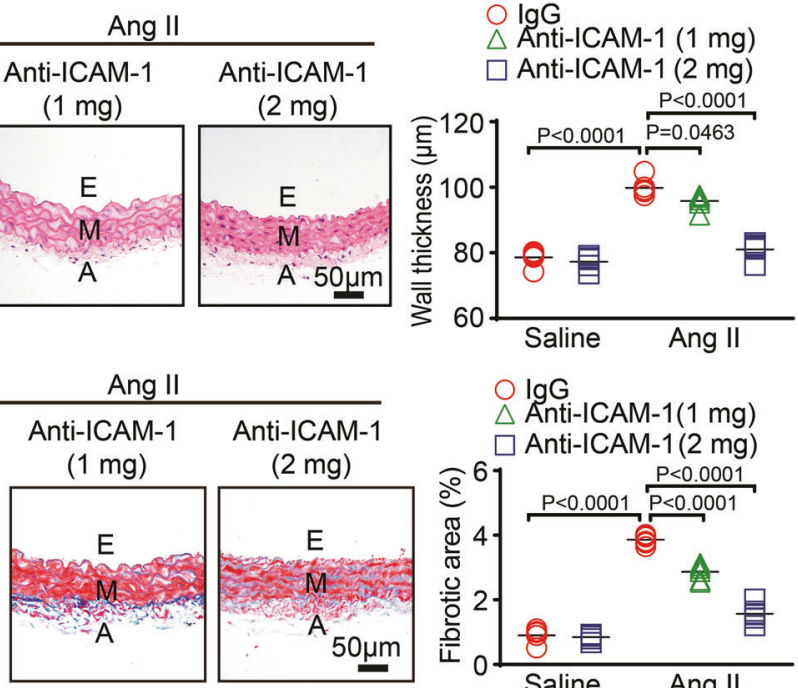

C
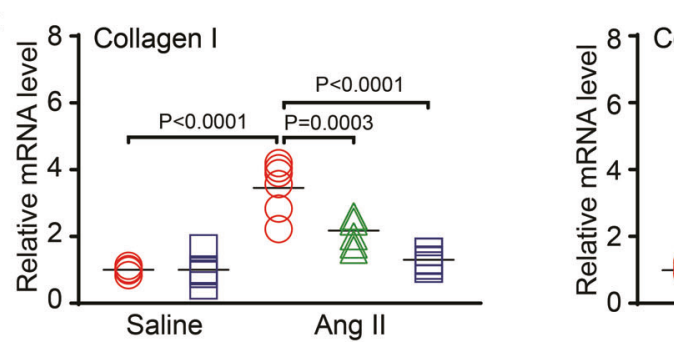

Fig. 3 The administration of the anti-ICAM-1 neutralizing antibody inhibits Ang II-induced vascular hypertrophy and fibrosis. a WT mouse was treated as described in Fig. 2. Images of H\&E staining of aortic in sections (left panel). Scale bar, $50 \mu \mathrm{m}$. Measurement of the aortic wall thickness (right panel, $n=6$ ). b Masson's trichrome

\section{Discussion}

This study is the first time to show that ICAM-1 upregulation is involved in Ang II-induced hypertension in mice. Following an Ang II infusion, ICAM-1 expression is increased in the aorta, which promotes monocyte attachment to EVs and subsequent transendothelial migration.
These changes induce the production of large quantities of proinflammatory cytokines and ROS, leading to vascular remodeling, dysfunction, and hypertension. Conversely, blockade of ICAM-1 significantly attenuates these effects (Fig. 7). Thus, these results provide novel evidence supporting a causal role for ICAM-1 in the pathogenesis of arterial hypertension. 

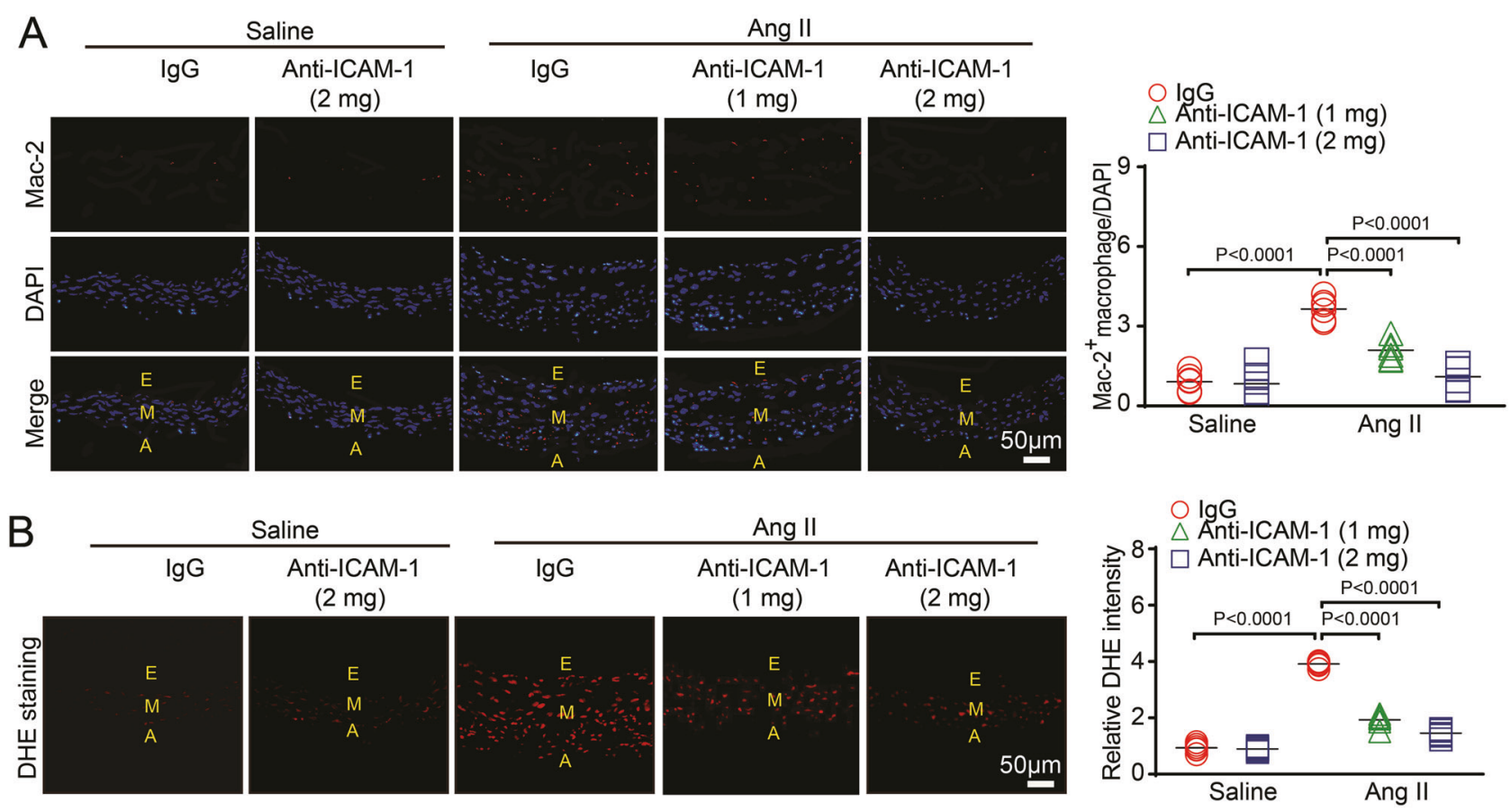

C $\bigcirc \lg G \triangle$ Anti-ICAM-1 (1 mg) $\square$ Anti-ICAM-1 (2 mg)
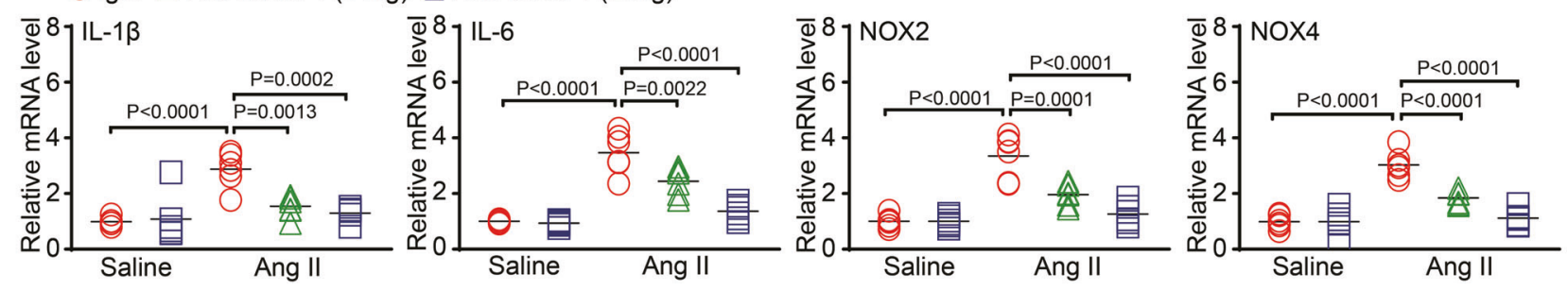

Fig. 4 The administration of the anti-ICAM-1 neutralizing antibody reduces Ang II-induced vascular macrophages recruitment and oxidative stress. a Mac-2 immunostaining in macrophages in aortic sections with an anti-Mac-2 antibody (left panel). Scale bar, $50 \mu \mathrm{m}$. Quantification of the number of Mac- $2^{+}$cells in each group (right, $n=6$ ).

b Dihydroethidium (DHE) staining of aortic sections (left panel). Scale bar, $50 \mu \mathrm{m}$. Quantification of the DHE intensity in each group (right panel, $n=6$ ). c Quantitative PCR analysis of IL-1 $\beta$, IL-6, NOX2, and NOX4 mRNA levels in each group
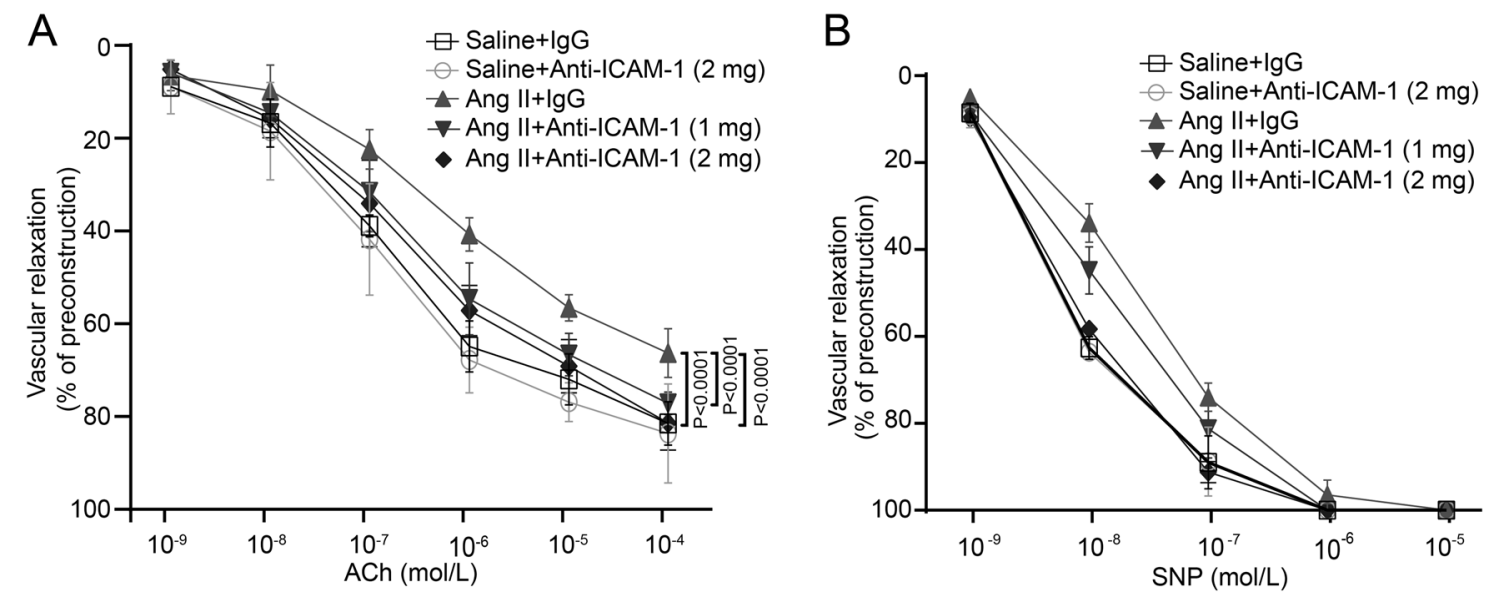

Fig. 5 Treatment with the anti-ICAM-1 neutralizing antibody improves Ang II-induced vascular dysfunction. a Aortic rings were isolated from IgG or anti-ICAM-1 ( 1 or $2 \mathrm{mg} /$ mouse/day)-treated WT mice subjected to 14 days of saline or Ang II infusions. Endothelium-

dependent relaxation in response to increasing doses of acetylcholine ( $n=6$ samples per group). b Endothelium-independent relaxation in response to increasing doses of sodium nitroprusside ( $n=6$ per group) 

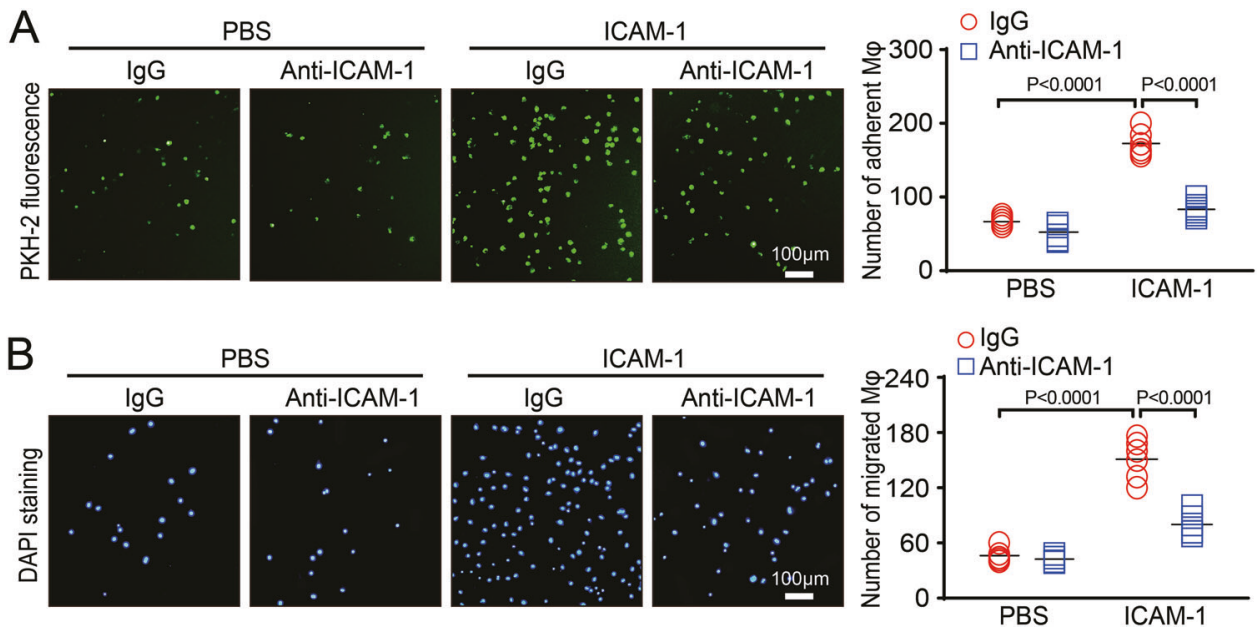

Fig. 6 Blockade of ICAM-1 function attenuates macrophages adhesion and migration. a Confluent HUVECs that had been treated with Ang II $(100 \mathrm{nM})$ or saline for $24 \mathrm{~h}$ were subsequently incubated with the isotype control IgG or anti-ICAM-1 neutralizing antibody for an additional $4 \mathrm{~h}$. Macrophages labeled with the PKH-2 fluorescent green dye were added to HUVECs at a macrophage-to-endothelial cell ratio of 10:1. Adherent cells were counted in six randomly chosen fields

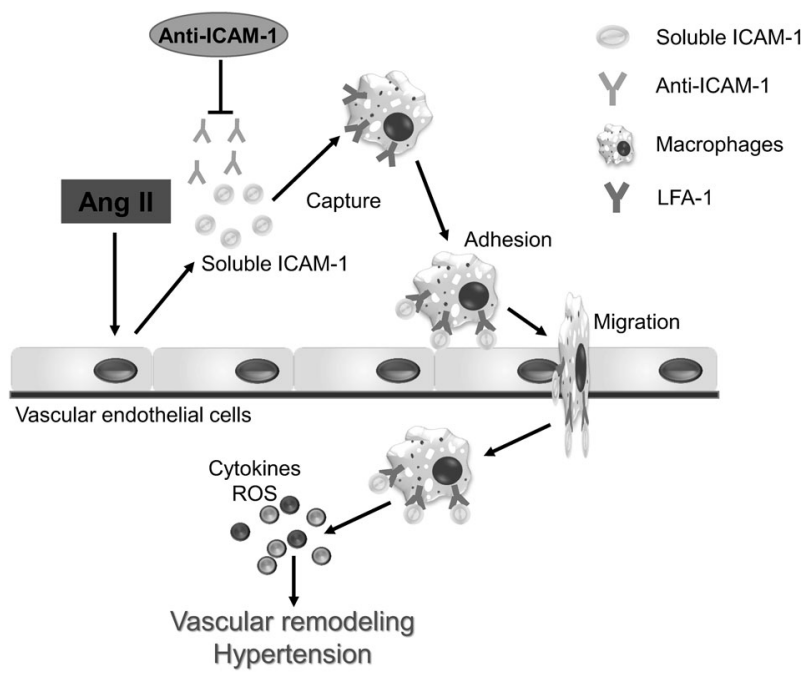

Fig. 7 A working model for ICAM-1 in the regulation of Ang IIinduced hypertension and vascular dysfunction

Based on accumulating evidence, inflammation is markedly involved in vascular fibrosis and dysfunction during the development of hypertension. Ang II increases the production of cytokines, chemokines, and CAMs, which initiate inflammatory pathways associated with ROS generation, and increase leukocyte adhesion to EVs and recruitment events [21, 22]. In addition to chemokines, adhesion molecules have the potential to regulate leukocytearterial interactions and are involved in mechanisms regulating leukocyte adhesion and trafficking [23]. Ang II using fluorescence microscopy (left panel). Quantification of the number of macrophages that adhered to HUVECs (right panel, $n=6$ ). b Macrophages isolated from WT mice were pretreated with the isotype control IgG or anti-ICAM-1 antibody for $4 \mathrm{~h}$, and then treated with PBS or ICAM-1 for $24 \mathrm{~h}$. Migration was assessed using a transwell assay. DAPI staining of the migrated macrophages $(\mathrm{M} \varphi)$ (left panel) and quantification (right panel, $n=6$ ). Scale bar, $100 \mu \mathrm{m}$

mediates the L-NAME-induced attachment of leukocytes to the arterial endothelium and induces the ICAM-1 expression in the arterial endothelium of rats, which was attenuated by the administration of the AT1 receptor antagonist losartan [24]. An Ang II treatment also upregulates ICAM-1 expression and stimulates soluble ICAM-1 secretion from cultured HUVECs via the AT1 receptor [10]. Moreover, the upregulation of ICAM-1 expression in the brain has been observed in ischemic animals and human patients with stroke [25, 26]. In addition, circulating levels of soluble ICAM-1, VCAM-1, and E-selectin are increased in older men with essential hypertension [27]. Interestingly, the inhibition of ICAM-1 reduces macrophage accumulation and TGF- $\beta$ expression, thereby attenuating cardiac fibrosis induced by pressure overload [11] and confirming a profibrotic role for ICAM-1 in the hypertensive heart. The present study extends previous findings by showing that an Ang II infusion significantly increases the levels of the ICAM-1 mRNA and protein in the aorta and serum of mice (Fig. 1), indicating that the production of soluble ICAM-1 may trigger the inflammatory response in the aorta. We used an anti-ICAM-1 neutralizing antibody that effectively reduces ICAM-1 levels in vivo in various experimental animal models, including models of vascular remodeling [28], cardiac fibrosis [11], and glomerulonephritis [29], to further investigate the causal role of ICAM-1 in hypertension. As expected, the administration of anti-ICAM-1 reduced the elevated blood pressure in a dose-dependent manner and attenuated the extent of vascular medial hypertrophy and fibrosis induced by Ang II (Figs. 2 and 3). 
Thus, ICAM-1 is involved in the mechanism regulating vascular remodeling and hypertension.

Leukocyte adhesion to activated EVs is an early event in various cardiovascular diseases, such as hypertension, cardiac remodeling, and atherosclerosis [30]. Based on the levels of CD14 and CD16, two subpopulations of monocytes were identified in human blood with different patterns of chemokine receptors and adhesion molecules on their surfaces [31], suggesting that these patterns may affect their adhesion to EVs and migration in the tissue. Monocytes are the main immune cells that regulate vascular dysfunction and hypertension induced by Ang II or high salt [3, 4]. The activation of monocytes/macrophages increases ROS production and contributes to the progression of vascular injury during hypertension [3]. Another important finding of this study is that the blockade of ICAM-1 function not only significantly inhibited the aortic infiltration of macrophages, ROS production, and the expression of IL-1 $\beta$, IL-6, NOX2, and NOX4 (Fig. 4), but also improved arterial endothelial dysfunction induced by Ang II (Fig. 5). Moreover, our in vitro experiments further confirmed that the blockade ICAM-1 function inhibited ICAM-1-induced macrophage adhesion to EVs and macrophage migration (Fig. 7). Thus, these data support a role for ICAM-1 in mediating vascular dysfunction through the regulation of cell adhesion, migration, and subsequent vascular oxidative stress. Inhibition of ICAM-1 in the early phase may be an effective strategy for preventing vascular dysfunction and hypertension in the later phase.

Although noninvasive tail-cuff method provides a useful tool in detecting blood pressure, it is incapable of continually measuring the blood pressure, and imposes substantial amounts of thermal and restraint stress to affect BP and heart rate. Thus, the effect of ICAM-1 on hypertension needs to further confirmed by radiotelemetry in the future.

In conclusion, ICAM-1 activity is essential for Ang IIinduced hypertension. Blocking ICAM-1 activity not only inhibits macrophage adhesion and migration, inflammation, and ROS production, but also attenuates vascular remodeling and subsequent vascular dysfunction after an Ang II infusion, indicating that ICAM-1 is a key molecule required for the initiation of macrophage-mediated hypertension. Therefore, therapeutic strategies targeting ICAM-1 might represent a promising approach for treating this disease. Future studies are needed to elucidate the initial mechanism responsible for inducing ICAM-1 expression in response to Ang II and confirm the effects of ICAM-1 in knockout mice.

Acknowledgements This work was supported by grants from the State Key Program of National Natural Science Foundation of China 81630009 (H.H.L.), Dalian High-Level Talents Innovation and Entrepreneurship Projects 2015R019 (H.H.L.), and the Chang Jiang Scholar Program of China T2011160 (H.H.L.).

\section{Compliance with ethical standards}

Conflict of interest The authors declare that they have no conflict of interest.

Publisher's note Springer Nature remains neutral with regard to jurisdictional claims in published maps and institutional affiliations.

\section{References}

1. Oparil S, Zaman MA, Calhoun DA. Pathogenesis of hypertension. Ann Intern Med. 2003;139:761-76.

2. Dharmashankar K, Widlansky ME. Vascular endothelial function and hypertension: insights and directions. Curr Hypertens Rep. 2010;12:448-55.

3. Wang L, Zhao XC, Cui W, Ma YQ, Ren HL, Zhou X, et al. Genetic and pharmacologic inhibition of the chemokine receptor CXCR2 prevents experimental hypertension and vascular dysfunction. Circulation. 2016;134:1353-68.

4. Wenzel P, Knorr M, Kossmann S, Stratmann J, Hausding M, Schuhmacher S, et al. Lysozyme M-positive monocytes mediate angiotensin II-induced arterial hypertension and vascular dysfunction. Circulation. 2011;124:1370-81.

5. Schiffrin EL, Touyz RM. From bedside to bench to bedside: role of renin-angiotensin-aldosterone system in remodeling of resistance arteries in hypertension. Am J Physiol Heart Circ Physiol. 2004;287:H435-446.

6. Hussain M, Awan FR. Hypertension regulating angiotensin peptides in the pathobiology of cardiovascular disease. Clin Exp Hypertens. 2018;40:344-52.

7. Gumbiner BM. Cell adhesion: the molecular basis of tissue architecture and morphogenesis. Cell. 1996;84:345-57.

8. Aricescu AR, Jones EY. Immunoglobulin superfamily cell adhesion molecules: zippers and signals. Curr Opin Cell Biol. 2007;19:543-50

9. Anbarasan C, Bavanilatha M, Latchumanadhas K, Ajit Mullasari S. ICAM-1 molecular mechanism and genome wide SNP's association studies. Indian Heart J. 2015;67:282-7.

10. Pastore L, Tessitore A, Martinotti S, Toniato E, Alesse E, Bravi MC, et al. Angiotensin II stimulates intercellular adhesion molecule-1 (ICAM-1) expression by human vascular endothelial cells and increases soluble ICAM-1 release in vivo. Circulation. 1999; 100:1646-52.

11. Kuwahara F, Kai H, Tokuda K, Niiyama H, Tahara N, Kusaba K, et al. Roles of intercellular adhesion molecule-1 in hypertensive cardiac remodeling. Hypertension. 2003;41(3 Pt 2):819-23.

12. Li N, Yang H, Wang M, Lü S, Zhang Y, Long M. Ligand-specific binding forces of LFA-1 and Mac-1 in neutrophil adhesion and crawling. Mol Biol Cell. 2018;29:408-18.

13. Tsakadze NL, Sen U, Zhao Z, Sithu SD, English WR, D'Souza SE. Signals mediating cleavage of intercellular adhesion molecule-1. Am J Physiol Cell Physiol. 2004;287:C55-63.

14. Komatsu S, Panes J, Russell JM, Anderson DC, Muzykantov VR, Miyasaka M, et al. Effects of chronic arterial hypertension on constitutive and induced intercellular adhesion molecule-1 expression in vivo. Hypertension. 1997;29:683-9.

15. Lee JR, Han JJ, Seo JW. Correlation between ICAM-1 and functional recovery of piglet myocardium with leukocyte-depleted reperfusion. Ann Thorac Surg. 2000;70:1531-5.

16. Cheng QL, Chen XM, Li F, Lin HL, Ye YZ, Fu B. Effects of ICAM-1 antisense oligonucleotide on the tubulointerstitium in mice with unilateral ureteral obstruction. Kidney Int. 2000;57:183-90. 
17. Wang L, Zhang YL, Lin QY, Liu Y, Guan XM, Ma XL, et al. CXCL1-CXCR2 axis mediates angiotensin II-induced cardiac hypertrophy and remodelling through regulation of monocyte infiltration. Eur Heart J. 2018;39:1818-31.

18. Wang X, Wang HX, Li YL, Zhang CC, Zhou CY, Wang L, et al. MicroRNA Let-7i negatively regulates cardiac inflammation and fibrosis. Hypertension. 2015;66:776-85.

19. Liao YH, Xia N, Zhou SF, Tang TT, Yan XX, Lv BJ, et al. Interleukin-17A contributes to myocardial ischemia/reperfusion injury by regulating cardiomyocyte apoptosis and neutrophil infiltration. J Am Coll Cardiol. 2012;59:420-9.

20. Takamatsu S. Naturally occurring cell adhesion inhibitors. J Nat Med. 2018;72:817-35.

21. Trott DW, Harrison DG. The immune system in hypertension. Adv Physiol Educ. 2014;38:20-24.

22. Harrison DG, Guzik TJ, Lob HE, Madhur MS, Marvar PJ, Thabet $\mathrm{SR}$, et al. Inflammation, immunity, and hypertension. Hypertension. 2011;57:132-40.

23. Galkina E, Ley K. Immune and inflammatory mechanisms of atherosclerosis (*). Annu Rev Immunol. 2009;27:165-97.

24. Nabah YN, Mateo T, Cerda-Nicolas M, Alvarez A, Martinez M, Issekutz AC, et al. L-NAME induces direct arteriolar leukocyte adhesion, which is mainly mediated by angiotensin-II. Microcirculation. 2005;12:443-53.

25. Bowes MO, Zivin JA, Rothlein R. Monoclonal antibody to the ICAM-1 adhesion site reduces neurological damage in a rabbit cerebral embolism stroke model. Exp Neurol. 1993;119:215-9.

26. Lindsberg PJ, Carpen O, Paetau A, Karjalainen-Lindsberg ML, Kaste M. Endothelial ICAM-1 expression associated with inflammatory cell response in human ischemic stroke. Circulation. 1996;94:939-45.

27. DeSouza CA, Dengel DR, Macko RF, Cox K, Seals DR. Elevated levels of circulating cell adhesion molecules in uncomplicated essential hypertension. Am J Hypertens. 1997;10 (12Pt1):1335-41.

28. Yasukawa $H$, Imaizumi T, Matsuoka $H$, Nakashima A, Morimatsu M. Inhibition of intimal hyperplasia after balloon injury by antibodies to intercellular adhesion molecule-1 and lymphocyte function-associated antigen-1. Circulation. 1997;95:1515-22.

29. Nishikawa K, Guo YJ, Miyasaka M, Tamatani T, Collins AB, Sy MS, et al. Antibodies to intercellular adhesion molecule 1/ lymphocyte function-associated antigen 1 prevent crescent formation in rat autoimmune glomerulonephritis. J Exp Med. 1993;177:667-77.

30. Huo Y, Ley K. Adhesion molecules and atherogenesis. Acta Physiol Scand. 2001;173:35-43.

31. Mosig S, Rennert K, Krause S, Kzhyshkowska J, Neunübel K, Heller $\mathrm{R}$, et al. Different functions of monocyte subsets in familial hypercholesterolemia: potential function of CD14+ CD16+ monocytes in detoxification of oxidized LDL. FASEB J. 2009;23:866-74. 